\title{
Minimally Invasive Isolated Tricuspid Valve Redo Surgery Has Better Clinical Outcome: A Single-Center Experience
}

\author{
Shixiong Wei, Lin Zhang, Huimin Cui, Tong Ren, Lianggang Li, Shengli Jiang \\ Department of Cardiovascular Surgery, Chinese PLA General Hospital, Beijing, 100853, China
}

\section{ABSTRACT}

Background: Reoperation for isolated tricuspid valve (TV) surgery is considered a high-risk procedure. The optimal surgical approach is controversial. We analyzed our experience with isolated TV redo surgery performed either through thoracoscopic approach (thoracoscopic group), right thoracotomy (thoracotomy group), or median sternotomy (sternotomy group).

Methods: We retrospectively analyzed all patients with previous cardiac surgery who underwent redo-TV procedure through thoracoscopic approach $(n=33)$, right lateral thoracotomy approach $(n=14)$, or sternotomy $(n=72)$.

Results: All patients successfully underwent elective surgery, with no intraoperative conversion or death occurring. $69 \%$ and $31 \%$ of patients received valve replacement and valvuloplasty, respectively. After operation, one patient in the sternotomy group received reoperation for bleeding, while another patient received valve replacement surgery 2 weeks after operation due to heart failure caused by valvuloplasty failure. No obvious complications occurred in the minimally invasive groups. The overall success rate of valve repair during 1-year follow-up was $99.2 \%$.

Conclusion: Minimally invasive, isolated TV surgery as reoperation can be safe and may improve clinical outcome.

\section{INTRODUCTION}

Tricuspid valve (TV) regurgitation has been identified as a major, previously widely underappreciated, risk factor for long-term mortality [Nath 2004; Pfannmüller 2012]. In particular, isolated redo TV procedures have been recognized as carrying an excessively high operative risk. Minimally invasive surgical approach by thoracoscopic techniques or via right lateral thoracotomy is well established for TV procedures [Casselman 2007; Bernal 2005]. Currently, isolated redo-TV operations with minimally invasive access are being used increasingly in our institution to treat progressive TV pathologic processes after previous cardiac operations. Here, we contribute our experience with special regard to the early

Received February 15, 2020; accepted April 28, 2020.

*Dr. Shixiong Wei and Dr. Lin Zhang contributed equally to this article. Correspondence: Shengli fiang, No.28 Fuxing Road, Haidian Distract, Beijing, 100853 China (e-mail: jiang_shengli301@163.com). postoperative course, complemented by a follow-up evaluation 1 year after surgery.

\section{MATERIALS AND METHODS}

Between January 2016 and December 2019, a total of 119 patients (46 males; mean age $47.6 \pm 18.5$ years) underwent isolated redo-TV surgery in our department. The underlying TV pathologies leading to surgery were severe tricuspid regurgitation due to annulus dilatation and/or leaflet restriction. All patients were operated on by the same surgeon. In all cases, access was achieved through one of thoracoscopic approach, right lateral thoracotomy, and median sternotomy. All patients were divided into thoracoscopic group $(\mathrm{n}=33)$, thoracotomy group $(\mathrm{n}=14)$, and sternotomy group $(\mathrm{n}=72)$ according to different surgical approaches. Ethics approval for this retrospective study was granted by the local ethics committee. Individual patient consent was not required.

\section{Surgical Technique}

Sternotomy Group. All patients were operated on with $\mathrm{CPB}$ either on a beating heart without cross-clamping the aorta or under cardioplegic arrest. Hypothermia was avoided and a nasopharyngeal temperature was kept at $35-37^{\circ} \mathrm{C}$ for the beating heart method; otherwise systemic moderate hypothermia was used $\left(30^{\circ} \mathrm{C}\right)$.

Thoracotomy Group. All of the patients were intubated with a double-lumen endotracheal tube and put in a right lateral decubitus position. Cushions were used to protect the brachial plexus and bone promontories. Right femoral artery cannulation was done for arterial inflow. A right anterolateral thoracotomy $10 \mathrm{~cm}$ in length through the fifth intercostal space was carried out and the right lung was deflated. After minimal pericardial dissection, bicaval venous cannulas were inserted through the right atrium for venous drainage. Then SVC and IVC were encircled with tapes. The left ventricle was decompressed through a vent placed from the right upper pulmonary vein. After going on CPB with normothermia (nasopharyngeal temperature, $35-37^{\circ} \mathrm{C}$ ), (flow rate: $2.587 \mathrm{~L} / \mathrm{min} /$ $\mathrm{m}^{2}$ ), mean systemic pressure was maintained at $60 \mathrm{mmHg}$. The heart was perfused through the aortic root and allowed to beat. When total CPB was begun by snaring the SVC and IVC with tapes, a right atriotomy was made. The patient was kept in Trendelenburg (head down tilt) position with continuous aortic root venting for air emboli prevention. De-airing procedures were continued until $\mathrm{CPB}$ was terminated. Transesophageal 
Table 1. Basic Characteristics of Patients

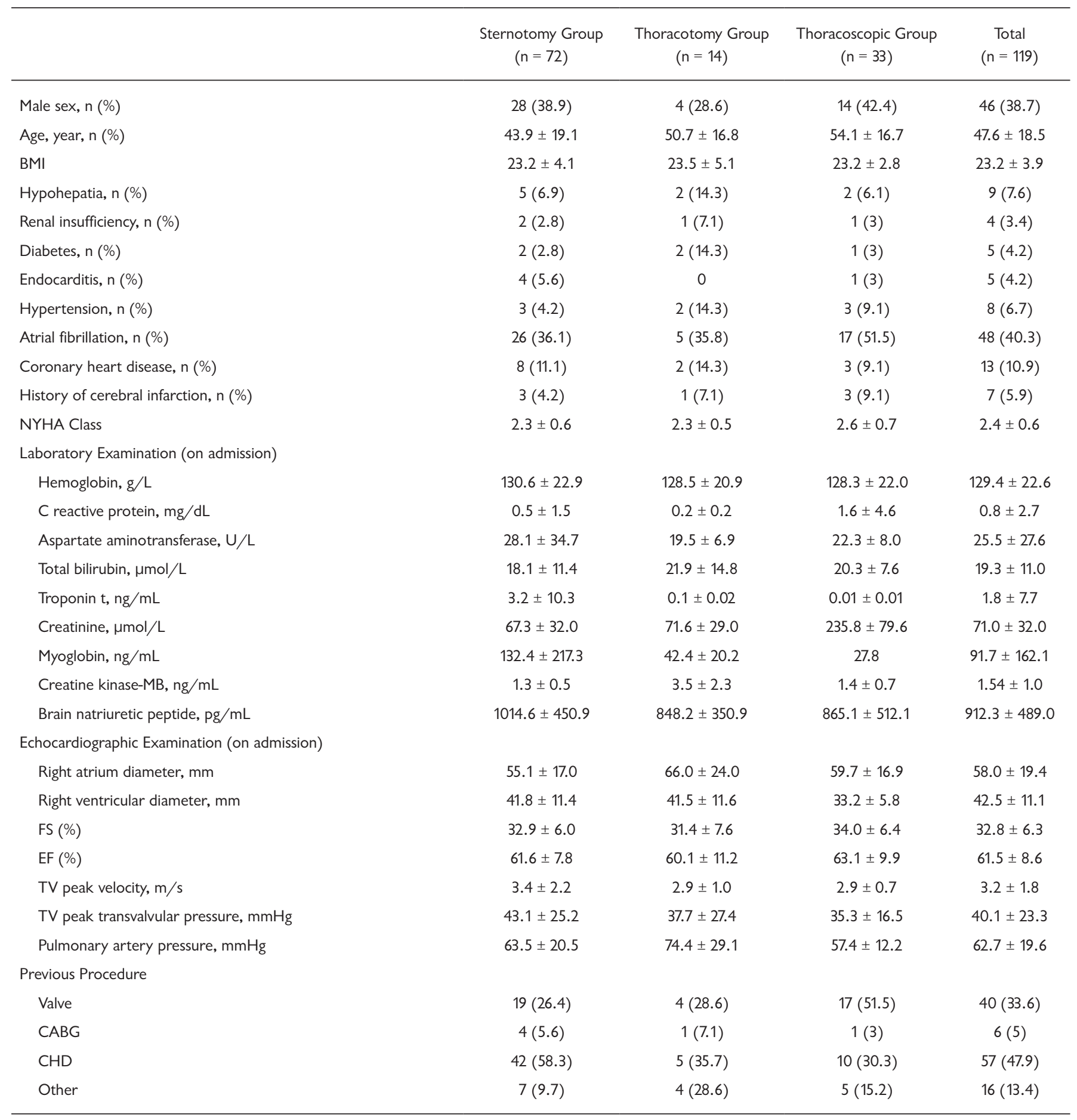

BMI indicates body mass index; $E F$, ejection fraction; $C A B G$, coronary artery bypass grafting; $C H D$, congenital heart disease.

echocardiography (TEE) was used to detect air bubbles before weaning from the $\mathrm{CPB}$. Tricuspid valve replacement was performed by standard fashion on a beating heart technique.

Thoracoscopic Group. All patients were examined preoperatively for adequate vascular access by an aorto-iliac and femoral vascular ultrasonography. Chest CT scan were performed routinely to rule out underlying pulmonary disease which may result in pleural thickening. In all patients, a double-lumen endotracheal tube was placed. External defibrillation pads were placed 
Table 2. Operative Outcomes

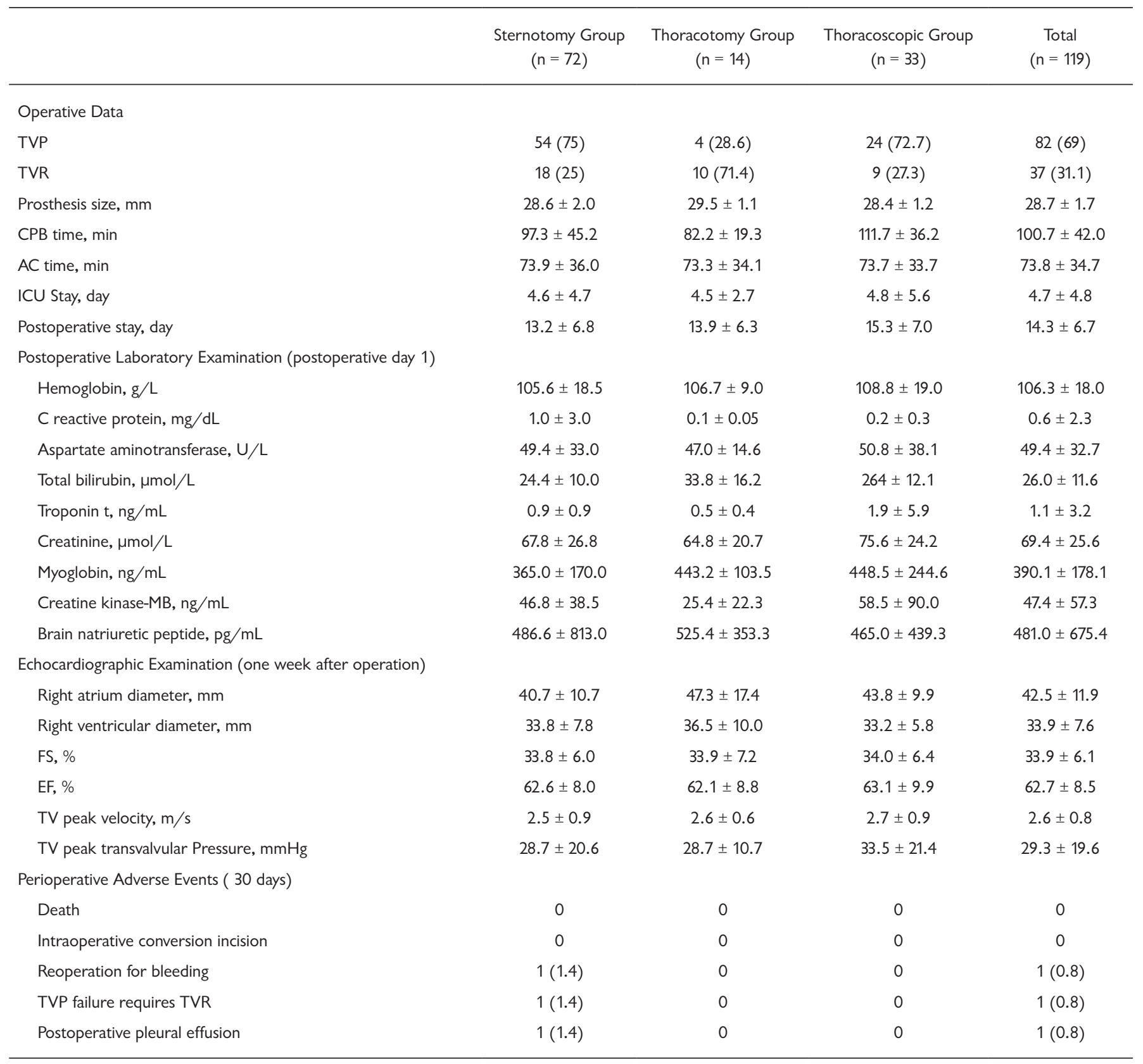

TVP indicates tricuspid valve plasty; TVR, tricuspid valve replacement; CPB, extracorporeal circulation; AC, aorta clamping.

in all patients. A minimal groin incision $(2-3 \mathrm{~cm})$ was made, and both femoral arterial and vein cannulations were directly performed using the Seldinger technique through two 5/0 Prolene purse strings. Arterial cannulation was all obtained through the femoral artery with an Medtronic (Minneapolis, MN, USA) cannula (15-18F according to the weight and arterial dimension). Venous return was routinely obtained with a femoral cannulation using a $21 \mathrm{~F}$ or $23 \mathrm{~F}$ Medtronic (Minneapolis, MN) cannula, which was delivered into the right atrium at about fifth intercostal space level. A less than 4-cm skin incision was made under the right nipple in men and in the right inframammary groove in women. A uniworking-port was made in the fourth intercostal space and the thoracic cage was embedded with CO2. Extensive lung adhesions may necessitate a change of strategy. Right atrium with pericardium was opened together. The inferior venal cannula tip was adjusted just below the Eustachian valve. Standard TV repair (TVP) or replacement (TVR) was performed on beating heart. 


\section{Follow-Up}

All patients were contacted by mail with a request to complete a comprehensive questionnaire. Patients who did not respond were contacted by our research personnel, and a telephone interview was conducted using the identical questionnaire, which was filled in by our research personnel. Finally, the general practitioners or next of kin were contacted directly. Hospital mortality is the mortality occurring within 30 days after the operation. The 1-year follow-up was $100 \%$ complete.

\section{Statistical Analysis}

Results are displayed in the standard format with continuous variables expressed as mean \pm standard deviation and categorical data as proportions. All statistical analysis was performed using SPSS statistical package, version 26.0 (SPSS, Chicago, IL).

\section{RESULTS}

Table 1 shows the preoperative and demographic data of the patient population separated by surgical access for isolated TV surgery. Patients in the thoracoscopic group were slightly older, included more males and atrial fibrillation with a worse NYHA Class. Patients in the thoracotomy group also had more hypohepatia, renal insufficiency, and hypertension. There was no significant difference in endocarditis, coronary heart disease, and history of cerebral infarction, admission laboratory examination, and echocardiography examination among the three groups of patients.

No intraoperative death or conversion of surgical procedures was observed. Table 2 shows the operative data and postoperative reexamination results. $69 \%$ of the patients $(n=82)$ received tricuspid valvuloplasty, while $71.4 \%$ of patients in the thoracotomy group $(n=10)$ received valve replacement surgery. Patients in the thoracoscopic group had slightly longer cardiopulmonary bypass time and aortic clamping time than patients in the other two groups. One patient in the sternotomy group received reoperation for bleeding 2 days after operation, while another patient received pleural effusion puncture 5 days after operation due to dyspnea caused by massive pleural effusion. In addition, one patient in the sternotomy group received valve replacement surgery two weeks after operation due to heart failure caused by valvuloplasty failure.

The 1-year follow-up rate was 100\% completed. All patients were successfully reexamined by echocardiography. No patients had obvious tricuspid regurgitation or stenosis again.

\section{DISCUSSION}

Despite improvements in the perioperative management, surgical procedures on the tricuspid valve still represent a therapeutic challenge. In-hospital mortality for this demanding patient cohort is reported to range between $13 \%$ and $26 \%$ [Minol 2017]. The limited outcome associated with isolated TV operations may be explained by the high rate of reoperation in this cohort, and furthermore by the high incidence of postoperative right heart failure and concomitant complications. Therefore, it must be evaluated whether a minimally invasive approach can be of benefit for this cohort of patients.

In 2001, Grossi and colleagues [Grossi 2001a] introduced their port-access approach for isolated tricuspid heart surgery and stated that this technique provided patients with significantly improved clinical outcomes. Right anterior minimally invasive thoracotomy and thoracoscopic techniques have become a well-established procedure in contemporary cardiac surgery [Minol 2017; Casselman 2003]. Postoperative morbidity and mortality rates are comparable to the results of the conventional approach via full sternotomy [Grossi 2001b]. At present, our center has successively carried out three surgical approaches (sternotomy, thoracotomy, and thoracoscopic) at the same time. In our experience, we found the two minimally invasive procedures to be highly operable and safe. In this study, $69.0 \%$ and $31.1 \%$ of patients underwent valve replacement and valvuloplasty, respectively.

The aorta clamping time of the three groups was similar. However, the extracorporeal circulation time of patients in the thoracoscopic group was slightly longer than that in the other two groups, which we think may be related to the learning curve and the higher proportion of valvuloplasty procedures.

The 1-year follow-up repair success rate of the two minimally invasive procedures was $100 \%$, while that of the sternotomy group was $99.2 \%$. Moreover, we only found postoperative complications in patients in the sternotomy group. As previously reported, the minimally invasive approach either via right lateral thoracotomy or thoracoscopic may be considered as advantageous over sternotomy approaches for redo TV procedures [Seeburger 2008]. Because of excellent exposure and insight on the TV and the subvalvular apparatus, a high repair rate can be achieved.

Our cohort displayed a 1 -year mortality rate of $0.8 \%$ overall, which may be in line with other series involving minimally invasive TV surgery [Ricci 2014], and appears to be quite encouraging when reports on TV surgery with full sternotomy are considered [Guenther 2008]. However, one has to consider that with increasing recognition of the minimally invasive approach, the standard of perioperative care has also improved significantly in recent years. Hence, contemporary results may not be entirely comparable to results of most studies analyzing the outcome of TV surgery via full sternotomy.

\section{Study Limitations}

This single-center retrospective study is mainly limited due to the small number of treated patients. Moreover, the cohort is quite heterogeneous. Furthermore, a longer followup time is required, especially to evaluate the clinical effects of the two minimally invasive procedures.

\section{Conclusion}

This study clearly indicates that minimally invasive TV surgery through either right anterolateral thoracotomy or thoracoscopic approach can be performed with results comparable to access via full sternotomy. 


\section{REFERENCES}

Bernal JM, Morales D, Revuelta C, Llorca J, GutierrezMorlote J, Revuelta JM. 2005. Reoperations after tricuspid valve repair. J Thorac Cardiovasc Surg 130:498-503.

Casselman FP, Van Slycke S, Wellens F, et al. 2003. Mitral valve surgery can now routinely be performed endoscopically. Circulation 108(Suppl 1):II48-54.

Casselman FP, La Meir M, Jeanmart H, et al. 2007. Endoscopic mitral and tricuspid valve surgery after previous cardiac surgery. Circulation 116:I270-5.

Grossi EA, Galloway AC, Ribakove GH, et al. 2001. Impact of minimally invasive valvular heart surgery: A case-control study. Ann Thorac Surg 71:807-10.

Grossi EA, LaPietra A, Ribakove GH, et al. 2001. Minimally invasive versus sternotomy approaches for mitral reconstruction: comparison of intermediate-term results. J Thorac Cardiovasc Surg 121:708-13
Guenther T, Noebauer C, Mazzitelli D, Busch R, Tassani-Prell P, Lange R. 2008. Tricuspid valve surgery: a thirty-year assessment of early and late outcome. Eur J Cardiothorac Surg 34:402-9, discussion 409.

Minol JP, Boeken U, Weinreich T, et al. 2017. Isolated tricuspid valve surgery: a single institutional experience with the technique of minimally invasive surgery via right minithoracotomy. Thorac Cardiovasc Surg 65:606-11.

Nath J, Foster E, Heidenreich PA. 2004. Impact of tricuspid regurgitation on long-term survival. J Am Coll Cardiol 43:405-9.

Pfannmüller B, Misfeld M, Borger MA, et al. 2012. Isolated reoperative minimally invasive tricuspid valve operations. Ann Thorac Surg 94:2005-10.

Ricci D, Boffini M, Barbero C, El Qarra S, Marchetto G, Rinaldi M. 2014. Minimally invasive tricuspid valve surgery in patients at high risk. J Thorac Cardiovasc Surg 147:996-1001.

Seeburger J, Borger MA, Falk V, et al. 2008. Minimal invasive mitral valve repair for mitral regurgitation: results of 1339 consecutive patients. Eur J Cardiothorac Surg 34:760-5. 